\title{
Relevant Research on the Development of Virtual Instrument Integrated Test System
}

\author{
Li Wang ${ }^{1,}$, , Hongxia Guo ${ }^{2}$ \\ ${ }^{1}$ Department of Energy Engineering, Yulin University, Yulin 719000, Shannxi, China \\ a278116312@qq.com
}

Keywords: virtual instrument; integrated test system; development and application; research Abstract. With the constant improvement of economic level in China and quick development of Internet technology, virtual instrument technology has been developed and applied rapidly. The genesis and application of virtual instrument technology is based on PC measurement technology. So this article will study the features and applications of virtual instrument test system, from its composition, design and development.

\section{Introduction}

The application of virtual instruments relies on computer technology. By combining computer and instrument hardware, a friendlier graphical interface can be offered to users. This interface is known as virtual front panel. Users can apply and control an instrument by operating the computer. Virtual instruments integrate computer resources, including A/D, numbers, and I/O more openly and clearly, analyze and process data uniformly and express graphical user interface (GUI). The genesis and application of virtual instruments marked the beginning of intelligent network software. Tests based on network and software will become a technical support that drives the reform and development in various fields.

\section{The Composition of Virtual Instrument}

Virtual instrument hardware system. Computer hardware platforms and measurement and control features constitute the hardware system of virtual instrument. A computer hardware platform is computers in various forms, which undertake the management of hardware and software resources in virtual instruments and provide a basis for virtual instrument hardware application. Different measurement and control features are realized by different hardware. At present, the most commonly-used is VXI bus technology and modular plug-in VXI instrument. Both of them can control the standard command language for instruments and provide a controllable programming environment for data use. DAQ and GPIB are combined and connected to computer hardware closely [1].

Virtual instrument software system. The software structure is an important component of instrument, which is divided into interface software, driver and software development environment, etc. See Figure 2 for details. An I/O interface can complete the data access of register unit and provide information for underlying software. Thus, it is open and virtualized to make the I/O interface the basis of system software [2].

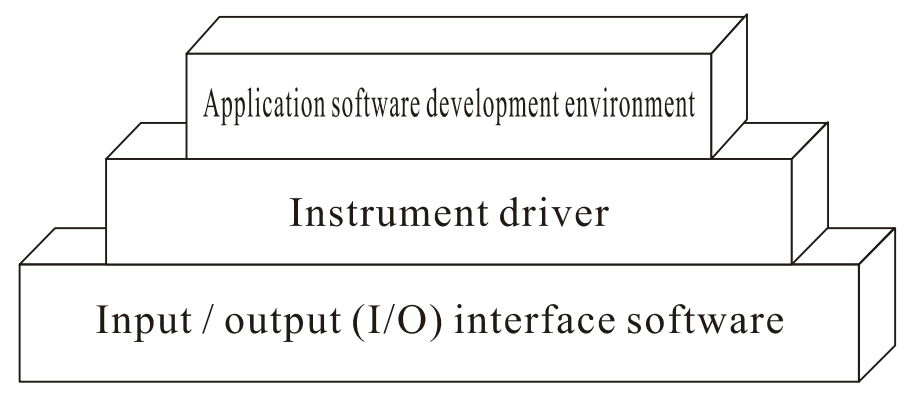

Figure 1 The Block Diagram of Virtual Instrument Software Structure 
The driver provides a set of functions for users to operate the instrument. Both the input and output are controlled by the driver, to provide a unified function format for interface software [3]. The application software is programmed by users. At present, the application software of virtual instruments has special open environments. One is text language development platform and the other is $\mathrm{VC}$ and $\mathrm{VB}$, etc. launched by NI company.

\section{The Design of Virtual Instrument Test System}

\subsection{The choice of virtual instrument platform}

Currently, virtual instruments have four standard platforms, i.e., VXI, PXI, GPIB and PC-DAQ. GPIB expands and extends the functions of instruments based on computer. PC-DAQ, however, uses industrial computer bus to support operation. But it has no bus performance. VXI and PXI platforms have their own advantages and disadvantages. When choosing them, we must consider complexity and budget. Small test systems can be chosen for laboratories. The system complexity is low. Large costs must be invested in initial construction, so PXI platform becomes the most suitable choice.

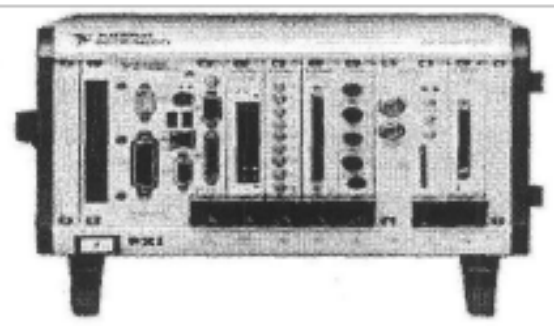

Figure 2 A Real Object of PXI System

As shown in the figure above, PXI is a virtual instrument platform. The bus is applied to computer bus basis similar to VXI. Corresponding instrument bus can be extended, to become a more standard test platform. PXI relies on PCI bus to a certain extent. $132 \mathrm{MB} / \mathrm{s}$ is the available bus throughput, which is higher than VME bus, and also makes the performance of PXI bus stronger [4].

2.2 Software structure

The software structure is applied to LabWindows/CVI development platform. This platform is fit for developers, who are accustomed to $\mathrm{C}$ language programming. It has flexible data acquisition, analysis and processing and more powerful $\mathrm{C} / \mathrm{C}++$ language features. It is highly integrated to development platform. LabWindows/CVI can program source code and 32-bit ANSIC and has multiple interactive features, such as debugging, compilation and link, etc., which make it a more comprehensive development platform. Thus, programming and debugging can proceed more quickly and conveniently [5]. Secondly, it has a visual GUI. LabWindows/CVI platform is generated and applied based on visualization technology and uses pop-up menus and program $\mathrm{C}$ code communication attributes. CVI generates source code files automatically.

\section{The Software Design of Test System}

\subsection{Master mode}

The master mode is responsible for parameter configuration and initialization, module scheduling and process control in the software system of test system. The functions of master module can be summed up as scheduling information and transmitting information, provide the only user access for the whole system, schedule and arrange work of many subsystems, thus realizing the modular design of the whole software and enhance module flexibility and software reusability, as shown in Tab. 1 below.

Tab. 1 Main Interface Parameters of Master Module

\begin{tabular}{ccc}
\hline Name & Type & Meaning \\
\hline RecNum & int & $\begin{array}{c}\text { Experiment record } \\
\text { number } \\
\text { tpTyroproject }\end{array}$ \\
Project confidence \\
\hline
\end{tabular}




\subsection{Project configuration}

To make the data and testing process proceed independently and make it more convenient to operate and use the system, we can set parameters related to laboratory and store them in a folder. A new project corresponds to a configuration file. Even if users are unfamiliar with system operation and have no idea about the system, they simply need to configure the project file to carry out an experiment. Such a design is very innovative. The experimental parameters are shown in Tab. 2 below.

Tab. 2 Interface Parameters of Project Configuration

\begin{tabular}{ccc}
\hline Name & Type & Meaning \\
\hline ProjectName & Char & Project name \\
Ch & Stauct & System channel \\
Reginfo & Stauct & Experimenter's \\
& & registration information \\
AdvApp & Stauct & Data acquisition and \\
& & configuration \\
\hline
\end{tabular}

\subsection{Channel calibration}

The so-called calibration means that the system builds a conversion relationship between physical quantity of measured object and acquired data objects. The accuracy of calibration directly affects the accuracy of data processing results. It is also an important premise for the starting of module and has a certain effect on data processing results and test results. Channel calibration must be done through system self-check and configuration module. The configuration must pass accurately in the first time. Otherwise, this calibration will make no sense. The module function is set to single channel calibration and partial channel calibration and offers recalibration feature [6]. The interface parameters of calibration module are shown in Tab. 3 .

Tab. 3 Interface Parameters of Calibration Module

\begin{tabular}{clc}
\hline Name & Type & Meaning \\
\hline LogChName & Char & Logical channel name \\
phyChName & Char & Physical channel name \\
Linear & Char & Fitting mode \\
ChUnit & Char & Physical unit \\
\hline
\end{tabular}

\section{Application Case}

An experiment is conducted on the vibration performance of mechanical structure of an underwater vehicle. The specific method is as follows:

\subsection{Broadband random test}

Broadband random signals contain all frequency component energy. It can excite the modality of experiment object at various stages and meet the requirements of experiment. White noise can simulate voltage signal output. The power spectrum is linear. We can amplify white noise power and vibrate randomly using an exciter. After basically getting stable, the dynamic frequency response test of system is finished. Convert into a power spectrum problem, record the output response signals and then process them accordingly. This method has many advantages, such as saving time, containing no highly-centralized frequency and realizing online test.

4.2 The way to adjust interference force

Converting white noise generated by a signal generator into sinusoidal signals is a way to adjust interference force. It is a steady state method and can test the inherent frequency of mechanical system. The current transferred from the power amplifier remains unchanged. The ejector pin system can act on a fixed amplitude and vibrate the mechanical system forcibly, according to electromagnetic interference force, which changes sinusoidally, thus improving the vibration frequency of exciter, measuring the amplitude and finding out the inherent frequency of mechanical system. The following table shows test results in the frequency band of 100HZ 900HZ: 
Tab. 4 The Way to Adjust Interference Force and Corresponding Amplitudes

Using the above two methods to measure the frequency of mechanical structure of the underwater

\begin{tabular}{|c|c|c|c|c|c|c|c|c|c|}
\hline 100 & 200 & 300 & 400 & 450 & 500 & 550 & 600 & 650 & 700 \\
\hline 0.024 & 0.003 & 0.0064 & 0.034 & 0.057 & 1.3 & 0.052 & 0.42 & 0.070 & 0.090 \\
\hline
\end{tabular}

\section{Conclusion}

This paper mainly analyzes from the composition, system design and development and practical application of virtual instruments, demonstrates that the virtual instrument integrated test system can realize various functions, with the help of computer technology and provide a technical support for the development of various fields. It has strong universality and practicality.

\section{Acknowledgements}

This work was supported by the Research of Gas Detection System Based on ZigBee and LabVIEW; and Municipal Science and Technology Bureau of Yulin City(Item number : 2015CXY-22-03)

\section{References}

[1] Ahmed F, Hawas Y E. An integrated real-time traffic signal system for transit signal priority, incident detection and congestion management[J]. Transportation Research Part $\mathrm{C}$ Emerging Technologies, 2015, 60:52-76.

[2] Aryanto K Y E, Broekema A, Oudkerk M, et al. Key Author Analysis in Research Professionals' Relationship Network Using Citation Indices and Centrality[J]. Procedia Computer Science, 2015, 57(1):606-613.

[3] Cartee J W. Significant aspects in the development of an integrated logistics support system in the Department of Defense -- The Single Manager Plan.[J]. Bju International, 2015, 116(4):502-3.

[4] Attanasio O P, Fernández C, Fitzsimons E O A, et al. Using the infrastructure of a conditional cash transfer program to deliver a scalable integrated early child development program in Colombia: cluster randomized controlled trial[J]. Bmj Clinical Research, 2014, 349(sep29 5):g5785-g5785.

[5] Dellaserra C L, Gao Y, Ransdell L. Use of integrated technology in team sports: a review of opportunities, challenges, and future directions for athletes.[J]. Journal of Strength \& Conditioning Research, 2014, 28(2):556-73. 